\title{
An overview of underwater sound generated by interparticle collisions and its application to the measurements of coarse sediment bedload transport
}

\author{
P. D. Thorne \\ National Oceanography Centre, Joseph Proudman building, 6 Brownlow Street, Liverpool L3 5DA, UK \\ Correspondence to: P. D. Thorne (pdt@noc.ac.uk)
}

Received: 6 June 2014 - Published in Earth Surf. Dynam. Discuss.: 9 July 2014

Revised: 20 November 2014 - Accepted: 21 November 2014 - Published: 23 December 2014

\begin{abstract}
Over the past 2 to 3 decades the concept of using sound generated by the interparticle collisions of mobile bed material has been investigated to assess if underwater sound can be utilised as a proxy for the estimation of bedload transport. In principle the acoustic approach is deemed to have the potential to provide non-intrusive, continuous, high-temporal-resolution measurements of bedload transport. It has been considered that the intensity of the sound radiated should be related to the amount of mobile material and the frequency spectrum to the size of the material. To be able to fully realise this use of acoustics requires an understanding of the parameters which control the generation of sound as particles impact. In the present work the aim is to provide scientists developing acoustics to measure bedload transport with a description of how sound is generated when particles undergo collision underwater. To investigate the properties of the sound generated, examples are provided under different conditions of impact. It is considered that providing an overview of the origins of the sound generation will provide a basis for the interpretation of acoustic data, collected in the marine environment for the study of bedload sediment transport processes.
\end{abstract}

\section{Introduction}

Quantifying bedload transport in rivers, estuarine and coastal environments is generally challenging due to the difficulties of obtaining accurate measurements. For measuring the bedload transport of coarse sediments, such as gravels and cobbles, a number of methodologies have been developed. Many measurements have utilised box or tray samplers, and this approach is still common today. Hubbell (1964), Engel and Lam Lau (1981), Ergenzinger and de Jong (2003), Bunte et al. (2008) and Holmes (2010), along with many others, have considered this technique. Some of the major shortcomings of this direct sampling method are the need for operators in the field, the limited range of conditions when measurements are possible, dangerous conditions during large floods, the impact on the flow introduced by the presence of the sampler, the lack of spatial/temporal resolution, the variable efficiency of samplers, the problems in obtaining continuous records and other difficulties specific to the particular samplers.
To circumvent some of these difficulties, alternative measurement technologies have been investigated. Dorey et al. (1975) investigated, with limited success, the feasibility of using sidescan sonar to track acoustically transponding pebbles. The tagging of gravel particles radioactively was developed by Crickmore et al. (1972) and applied with success to monitoring the movement of gravel. Reid et al. (1984) utilised artificial pebbles constructed with a ferrite rod at its centre and deployed them in a brook. Particle mobility was detected using an electromagnetic sensing system installed in the bed and transport compared with the bed shear. Geophones and particle impacts on pipes, columns and plates are now commonly used to estimate coarse gravel transport (Turowski and Rickenmann, 2009; Gray et al., 2010; Rickenmann et al., 2012). In recent years acoustic Doppler velocity profiles, ADCPs, have been used to measure apparent bedload velocity using a combination of ADCP bottom tracking velocity and boat velocity derived from differential global positioning systems, DGPSs (Rennie and Church, 2010). Re- 
cently the measurement of seismic noise near rivers has been investigated to assess bedload transport (Burtin et al., 2011; Tsai et al., 2012). A workshop on contemporary methodologies for the monitoring of bedload transport has recently been published online by Rickenmann et al. (2013).

Another approach adopted, and the one focussed upon here, has been to monitor the movement of gravel by recording the acoustic sediment-generated noise, SGN, arising from particle-particle collisions as bedload transport occurs. Observations of this type have continued to be reported over the past 5 decades (Bedeus and Ivicsics, 1963; Johnson and Muir, 1969; Tywoniuk and Warnock, 1973; Jonys, 1976; Richards and Milne, 1979; Thorne et al., 1984; William et al., 1989; Mason et al., 2007; Barton et al., 2010; Camenen et al., 2012; Basset et al., 2013). The appeal of the acoustic approach is that it offers the potential to obtain, with very little interference with the state of the bed and the flow, the initiation of particle movement, continuous temporal records, sub-second assessment of mass transport rates and estimates of mobile particle size.

To be able to utilise and interpret SGN with any degree of confidence requires an understanding of the sound source generation. The source arises from the impact of two or more particles as interparticle collisions occur as the bed becomes mobile and bedload transport occurs. The generation of sound by impacting bodies has primarily been examined in air, usually by parties interested in machine noise emissions (Banerji, 1916, 1918; Koss and Alfredson, 1973; Koss, 1974a, b; Akay and Hodgson, 1978a, b; Akay, 1978). It is this work which was adapted for the study of acoustic radiation by colliding bodies underwater (Thorne and Foden, 1988; Thorne, 1990). The source of radiation has been labelled rigid body radiation due to the origin of the pressure disturbance being generated by the acceleration of the body, rather than due to the natural modes of vibration of the body (Koss and Alfredson, 1973). To solve the problem, each sphere is treated as an independent source which generates a transient that can be described by an impulse solution convolved with the acceleration time history during the collision. The impact process is assumed to be elastic so that a Hertzian acceleration description can be employed (Goldsmith, 1960). The sound field is then obtained by summing the transients radiated from each sphere with due allowance for the time difference for the sound to propagate from each sphere to the field measurement point.

The use of SGN to acoustically measure bedload transport and the underlying theory of rigid body radiation is distributed among acoustic, geological, hydraulic, geophysical and sedimentological journals. The aim of the present work is to bring together an overview of SGN and its underlying theoretical basis, in a form which scientists interested in using acoustics for bedload transport would find useful. Here the main solutions from rigid body radiation analysis are simplified to make the topic more accessible and the outputs focussed more closely than previously on bedload transport.

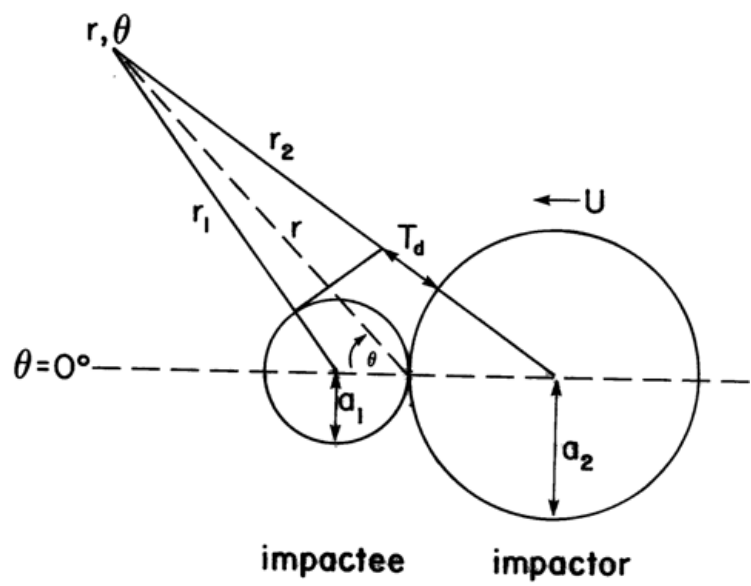

Figure 1. Geometry for the theory. The impactor of radius $a_{2}$ collides with velocity $U$ and with the impactee of radius $a_{1} . \theta$ is the angle between the line of sphere movement and the direction to the field point, and $r$ is the range to the field point. $T_{\mathrm{d}}$ is the difference in the arrival time of the sound radiated by the impactor relative to the impactee.

Therefore, using the rigid body radiation theory, initial calculations have been carried out to elucidate the origins of the structure of the radiated signal in the time and frequency domain. To investigate how changes in impact parameters affected the time domain signal and the frequency spectrum, a series of calculations were conducted. The intention of this work was to provide broad illustrations of the radiated signal response due to variation in sphere collision impact parameters. Some general features are identified and considered in the light of using SGN for the measurement of bedload transport. Some modelling of multiple impacts is presented as an analogy to the type of data that may be collected in a coastal or riverine environment. It is hoped that the present overview will stimulate further interest in SGN by making the acoustic analysis more straightforward and by illustrating its potential capability.

\section{Theory for the underwater sound generated by impacting spheres}

The background theory for impacting spheres in water was developed in Thorne and Foden (1988), and only the results from the theoretical analysis are presented here. The geometry for the theory is given in Fig. 1. When solid elastic spheres collide, the main source of sound generation is due to the rigid body radiation associated with the acceleration of the impactee of radius $a_{1}$ and the deceleration of the impactor of radius $a_{2}$ following impact. These produce sound waves which radiate from the spheres into the water. To simplify the analysis presented here, the first conditions assumed is $\rho_{\mathrm{s}} / 2 \rho_{\mathrm{o}} \ll 1$, where $\rho_{\mathrm{s}}$ is the density of spheres and $\rho_{\mathrm{o}}$ the density of water. This condition is not strongly adhered to 
for marine gravels with densities of the order of $2500 \mathrm{kgm}^{-3}$; however it considerably reduces the complexity of the time domain solution and simply leads to an overestimate of the signal levels by 10-20\%. The second condition is $r / a \gg 1$, where $r$ is the distance from the impacting spheres to the location at which the radiated sound is observed and $a$ is the radius of the spheres; this condition can generally be readily adhered to. The pressure, $P_{\mathrm{S}}(t)$, in the time domain for a single particle undergoing Hertzian impact (Goldsmith, 1960), with a half sine wave acceleration profile (Koss and Alfredson, 1973), can then be expressed as the convolution integral of the radiated pressure due to a unit impulse acceleration and the acceleration time history (Thorne and Foden, 1988); the solution is given by

$$
\begin{aligned}
& 0 \leq t \leq t_{\mathrm{O}} \\
& P_{\mathrm{S}}(t)=P_{\text {to }}\left\{\left(2 \xi^{2}-1\right) \cos \pi \tau+2 \xi \sin \pi \tau-\left[\left(2 \xi^{2}+1\right)\right.\right. \\
& \left.\left.\sin \pi \xi \tau+\left(2 \xi^{2}-1\right) \cos \pi \xi \tau\right] e^{-\pi \xi \tau}\right\} \\
& t>t_{\mathrm{O}} \\
& P_{\mathrm{S}}(t)=P_{\text {to }}\left\{\left[\left(1-2 \xi^{2}\right) \cos \pi \xi(\tau-1)-\left(2 \xi^{2}+1\right)\right.\right. \\
& \sin \pi \xi(\tau-1)] e^{-\pi \xi(\tau-1)}-\left[\left(2 \xi^{2}+1\right) \sin \pi \xi \tau\right. \\
& \left.\left.+\left(2 \xi^{2}-1\right) \cos \pi \xi \tau\right] e^{-\pi \xi \tau}\right\}
\end{aligned}
$$

where $t_{o}$ is the duration time of the collision, $\xi$ is a nondimensional parameter relating the collision time to the sphere size, $\tau$ is a normalised time parameter and $P_{\text {to }}$ is a pressure amplitude scaling factor; these four parameters are defined below. The pressure wave, $P_{\mathrm{p}}(\mathrm{t})$, from the impactor and the impactee pair, is the combined pressure wave radiated from each sphere with due allowance for the time delay of the impactor signal relative to the impactee signal as given below:

$P_{\mathrm{p}}(t)=P_{\mathrm{S}}\left(t, \mathrm{a}_{1}\right)+P_{\mathrm{S}}\left[\left(t-T_{\mathrm{d}}\right), a_{2}\right]$.

$T_{\mathrm{d}}$ is the difference in the arrival time of the sound radiated by the impactor relative to the impactee. Equation (1) provides the time domain waveform for the sound radiated when one sphere impacts with another. A number of parameters are defined below:

$$
\begin{aligned}
& P_{\text {to }}=\frac{a_{j} A_{j} \rho_{\mathrm{o}} \mathrm{cU} \cos \theta}{2 r\left(4 \xi^{4}+1\right)} \\
& t_{\mathrm{o}}=4.53\left[\left(\frac{1-\sigma_{1}^{2}}{E_{1} \pi}+\frac{1-\sigma_{2}^{2}}{E_{2} \pi}\right)\left(\frac{m_{1} m_{2}}{m_{1}+m_{2}}\right)\right]^{0.4} \\
& \left(\frac{a_{1}+a_{2}}{U a_{1} a_{2}}\right)^{0.2}, \\
& A_{1}=2 m_{2} /\left(m_{1}+m_{2}\right), A_{2}=-\left(m_{1} / m_{2}\right) A_{1}, \\
& \xi=c t_{\mathrm{o}} / \pi a_{j}, \tau=t / t_{\mathrm{o}} .
\end{aligned}
$$

Here $m$ is the sphere mass, $E$ Young's modulus, $\sigma$ Poisson's ratio for the spheres and subscripts (1) and (2) refer to the impactee and the impactor; $j=1$ or $2 . T_{\mathrm{d}} \approx a_{1}(1+\pi / 2) / c$ for $\theta=0$ and $T_{\mathrm{d}} \approx 2 \operatorname{acos} \theta / c$ when $a_{1}=a_{2}$ and $\theta>0$, where $\theta$ is the angle between the line of sphere movement and the direction to the field point, $c$ is the velocity of sound in water, $U$ is the impact velocity, $r$ is the range to the field point and $t$ is time. $A_{j} U \pi /\left(2 t_{\mathrm{o}}\right)$ is the maximum acceleration of each sphere. Polar co-ordinates were selected for the analysis because of the axial symmetry of the radiated sound about the direction of polar angle, $\theta$, in the azimuth direction.

A similar expression can be obtained for the frequency spectrum when two spheres collide by using the time convolution theorem. This provides the spectrum for the collision from the product of the Fourier transform of the radiated pressure due to a unit impulse acceleration, with the Fourier transform of the acceleration time history; this results in the expression given below (Thorne and Foden, 1988):

$$
\begin{aligned}
& P_{\mathrm{s}}(f)=P_{\mathrm{fo}} \frac{1+e^{-i \pi \varepsilon}}{1-\varepsilon^{2}} \frac{\xi a_{j}+i r \varepsilon}{2 \xi^{2}-\varepsilon^{2}+2 i \xi \varepsilon}, \\
& P_{\mathrm{p}}(f)=P_{\mathrm{s}}\left(f, a_{1}\right)+P_{\mathrm{s}}\left(f, a_{2}\right) e^{-i \omega T_{\mathrm{d}}},
\end{aligned}
$$

where

$$
\begin{aligned}
& P_{\mathrm{fo}}=\frac{a_{j} A_{j} \rho_{\mathrm{o}} \mathrm{cU} \cos \theta}{2 \mathrm{r}^{2} \omega_{\mathrm{o}}}, \\
& \varepsilon=f / f_{\mathrm{o}}, \quad f_{\mathrm{o}}=1 / 2 t_{\mathrm{o}}, \quad \omega_{\mathrm{o}}=2 \pi f_{\mathrm{o}}, \quad i=\sqrt{-1} .
\end{aligned}
$$

To illustrate the sound generated when one particle impacts with another, Fig. 2 shows how the structure of the pressure wave is formed. The predicted underwater rigid body radiation for two spheres impacting was computed using Eqs. (1) and (2). Glass sphere properties were chosen for the calculations to represent marine shingle as both materials are often composed of silicates. For the calculation presented in Fig. 2, the velocity of sound and the density of water were respectively taken to be $1480 \mathrm{~ms}^{-1}$ and $1000 \mathrm{kgm}^{-3}$, for the colliding spheres $a_{1}=a_{2}=0.02 \mathrm{~m}, \rho_{\mathrm{s}}=2500 \mathrm{kgm}^{-3}$, $U=0.3 \mathrm{~ms}^{-1}, r=0.3 \mathrm{~m}, \theta=0^{\circ}, E=7.10^{10} \mathrm{Nm}^{-2}$ and $\sigma=0.2$ (Kaye and Laby, 1986). In Fig. 2a, the time series solution is presented. The first signal to arrive is from the accelerating impactee; this consists initially of a compressive peak from the surface of the sphere closer to the receiver, followed by a rarefaction from the rear surface. After a delay, $T_{\mathrm{d}}$, the signal from the impactor arrives. This is phase-inverted due to the impactor decelerating in the direction towards the receiver, thereby giving initially a rarefaction followed by a compression peak. The total signal from the two spheres can be seen to have an "M" waveform. The spectrum, obtained using Eq. (2), can be seen in Fig. 2b. The spectrum is oscillatory in form peaking at $f \approx 1.7 f_{\mathrm{o}}$, with nulls at $f=(2 n+1) f_{\mathrm{o}}$, where $\mathrm{n}$ is an integer and with the spectrum reducing to negligible values above $7 f_{\mathrm{o}}$. The oscillations in the spectrum are associated with the term arising from the Fourier transform of the half sinusoidal acceleration profile. Software to calculate the 

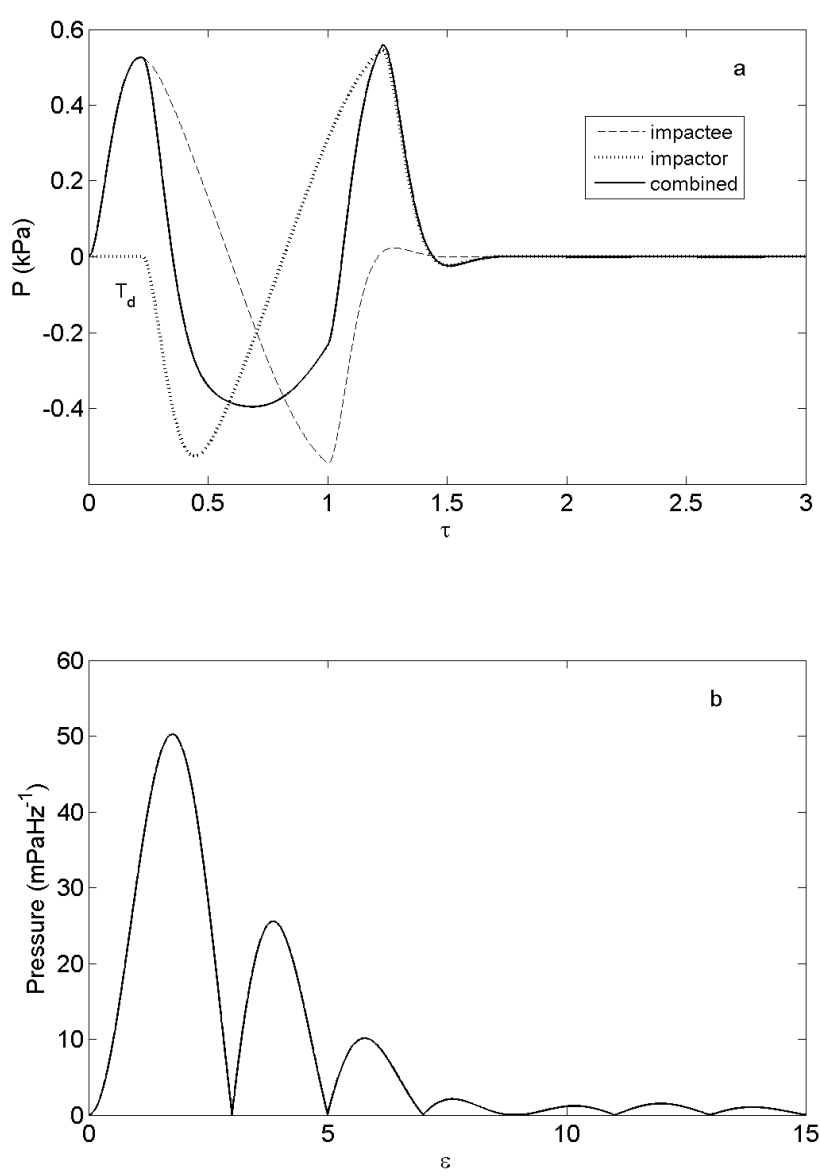

Figure 2. Calculations for two glass spheres of the same size impacting with radius $a=0.02 \mathrm{~m}$ and impact velocity $U=0.3 \mathrm{~ms}^{-1}$. (a) The time domain solution using Eq. (1) for the pressure, $P$, with normalised time $\tau=t / t_{\mathrm{O}}$ and (b) the frequency domain solution using Eq. (2) for the spectral pressure with normalised frequency $\varepsilon=f / f_{\mathrm{o}}$.

impact radiation is given at http://noc.ac.uk/using-science/ products/software/csr-acoustic-inversions Program glass_sphere_impact_paper.m. This software defines all the terms in Eqs. (1) and (2) and allows users to investigate the properties of the sound radiated as parameters are changed for the impacting spheres.

\section{Prediction for the radiated sound and comparisons with laboratory observations}

\section{1 (i) Single particle pair impacts}

The aim here is to provide insight into the structure of the rigid body radiation field as impact parameters were varied. Therefore a number of calculations for the time domain waveform and the frequency spectrum were carried out. Initially spheres of equal size were considered with radii of $0.005,0.015$ and $0.05 \mathrm{~m}$. These spheres were chosen to represent a broad range of particle sizes from fine pebbles to cob- bles. This was considered to be representative of the common sizes of materials on which the acoustic technique would be used for bedload measurements. The results are considered as indicative of the signals that would arise from individual particle pair impacts in coarse sediment bedload transport. For the calculations Eqs. (1) and (2) were evaluated with all the others parameter having the same values as used to obtain Fig. 2. Figure 3 shows the results of the computations. Considering the time domain waveforms shown in plots $3 \mathrm{a}-$ $\mathrm{c}$, it can be observed that both the duration and amplitude, $P_{\mathrm{p}}$ $(t)$, of the waveform increase with sphere size. For spheres of equal size $A_{2}=-A_{1}=1, \xi$ is constant, and therefore it is the linear dependence of $P_{\text {to }}$ on $a$ which produces the increase in amplitude with sphere size. The duration of the waveform is controlled by $t_{\mathrm{o}}$ and $T_{\mathrm{d}}$, and for equally sized spheres both are a linear function of $a$. Therefore the waveform duration linearly increases with sphere size. For the spectra shown in plots $3 \mathrm{~d}-\mathrm{f}$ the spectrum shifts to lower frequencies and increases in amplitude as sphere size increases. The shift to lower frequencies is due to the inverse relationship between $f_{\mathrm{o}}$ and $t_{\mathrm{o}}$ and hence $f_{\mathrm{o}} \alpha 1 / a$. For the spectral amplitude, $P_{\mathrm{p}}(f)$, it is a function of $a / \omega_{\mathrm{o}}$, with $\omega_{\mathrm{o}}$ being dependent on $1 / a$ through $t_{\mathrm{o}}$; hence $P_{\mathrm{p}}(f)$ is proportional to $a^{2}$ as seen in the plots. In Thorne and Foden (1988) a number of comparisons were made between predictions and measurements of impacting steel spheres underwater; the results supported the rigid body impact model. Two cases of glass spheres impacting underwater were also made, and the results for $a_{1}=a_{2}=0.015 \mathrm{~m}$ case are compared with predictions in Fig. $3 b$ and e, where it can be seen that the predictions from rigid body radiation compare very favourably with the observations.

In the marine environment particles of different size will generally be impacting, and it is therefore interesting to examine this case. To assess the radiated sound field from spheres of different size impacting, calculations were carried out using the same parameters as used for Fig. 3, but with the individual impacting spheres having a different radius. The results are presented in Fig. 4 and can be directly compared with the plots in Fig. 3. In the frequency spectrum plots in Fig. 4 the vertical solid and dashed lines respectively show the location of the spectral peak for the same size spheres impacting with radii $a_{1}$ and $a_{2}$. For the case of $a_{1}=0.005 \mathrm{~m}$ and $a_{2}=0.015 \mathrm{~m}$ the time domain waveform and frequency spectrum are given in plots $4 \mathrm{a}$ and $4 \mathrm{~d}$. Relative to the case of $a_{1}=a_{2}=0.005 \mathrm{~m}$ shown in Fig. $3 \mathrm{a}$ and $\mathrm{d}$, the time domain signal is $50 \%$ longer in duration and $25 \%$ higher in amplitude, while the location of the peak frequency in the spectrum has reduced by $20 \%$, lying between the two vertical lines, and with a spectral amplitude which has doubled. Comparing the results for $a_{1}=0.005 \mathrm{~m}$ and $a_{2}=0.015 \mathrm{~m}$ with $a_{1}=a_{2}=0.015 \mathrm{~m}$ in Fig. $3 \mathrm{~b}$ and e, the converse is the case, with the duration and amplitude of the time domain waveform being reduced and the location of the peak frequency in the spectrum doubled and the spectral amplitude 

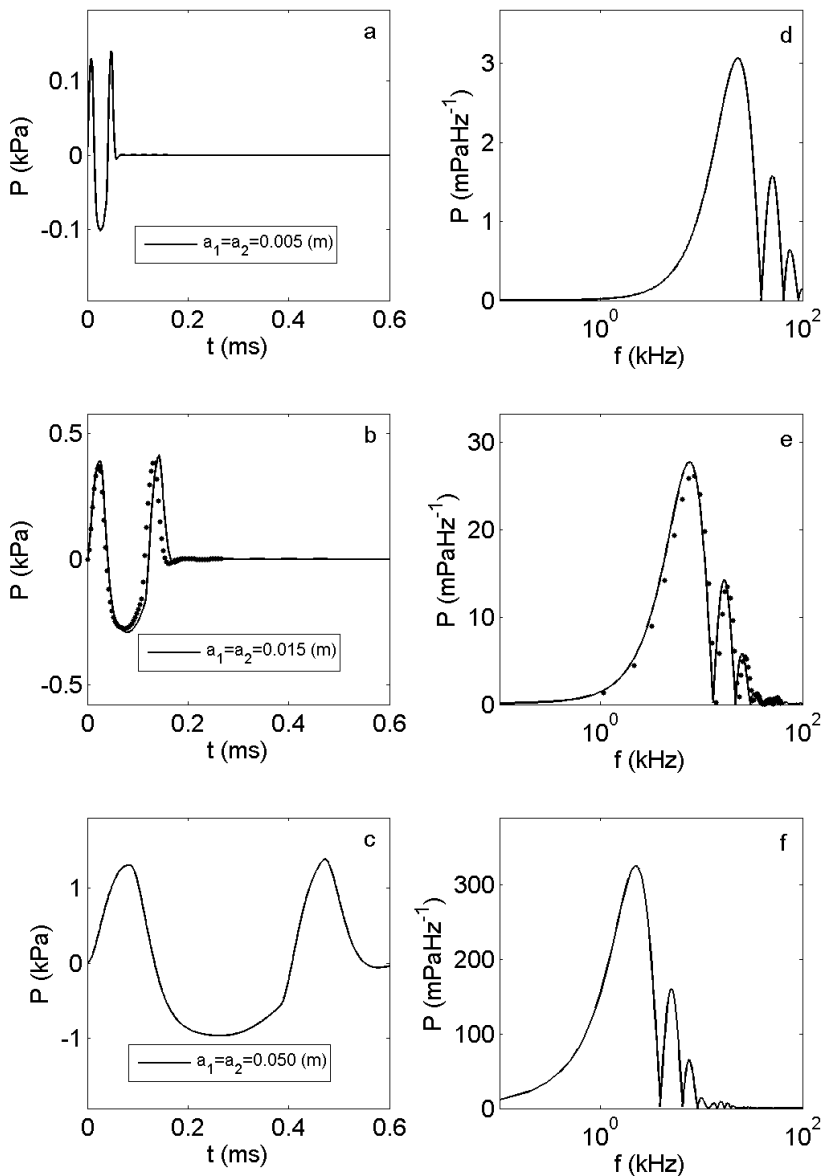

Figure 3. Calculations for glass spheres of the same size impacting as the sphere size was increased. (a)-(c) Time domain waveform calculated using Eq. (1) and (d)-(f) frequency spectrum calculated using Eq. (2). Measurements (•) are from Thorne and Foden (1988).

significantly lower. Colliding spheres of different size therefore have an admixture of the radiated sound properties of the accelerating impactee, $a_{1}$, and the decelerating impactor, $a_{2}$. Plots $4 \mathrm{~b}, \mathrm{e}, \mathrm{c}$ and $\mathrm{f}$ further illustrate this admixture when compared with results presented in Fig. 3. Such admixtures will almost certainly be present in riverine bedload transport as the bed will invariably by composed of a broad range of particle sizes.

To assess how changes in the impact velocity, $U$, and the field point angle, $\theta$, affected the time domain waveform and the frequency spectrum, a number of calculations were carried out, and the results are presented in Fig. 5. For these calculations $a_{1}=a_{2}=0.015 \mathrm{~m}$, and apart from the changes in $U$ and $\theta$ all other parameters were the same as used for the calculations shown in Fig. 2. As shown in plots 5a and $\mathrm{b}$, an increase in $U$ has a marginal influence on the duration of the time domain waveform and the frequency spectrum covered, with the main response being an increase in signal amplitude. The former is due to the weak dependence of $t_{\mathrm{o}}$ on $U^{-0.2}$, while inspection of Eqs. (1) and (2), for $\xi>1$,
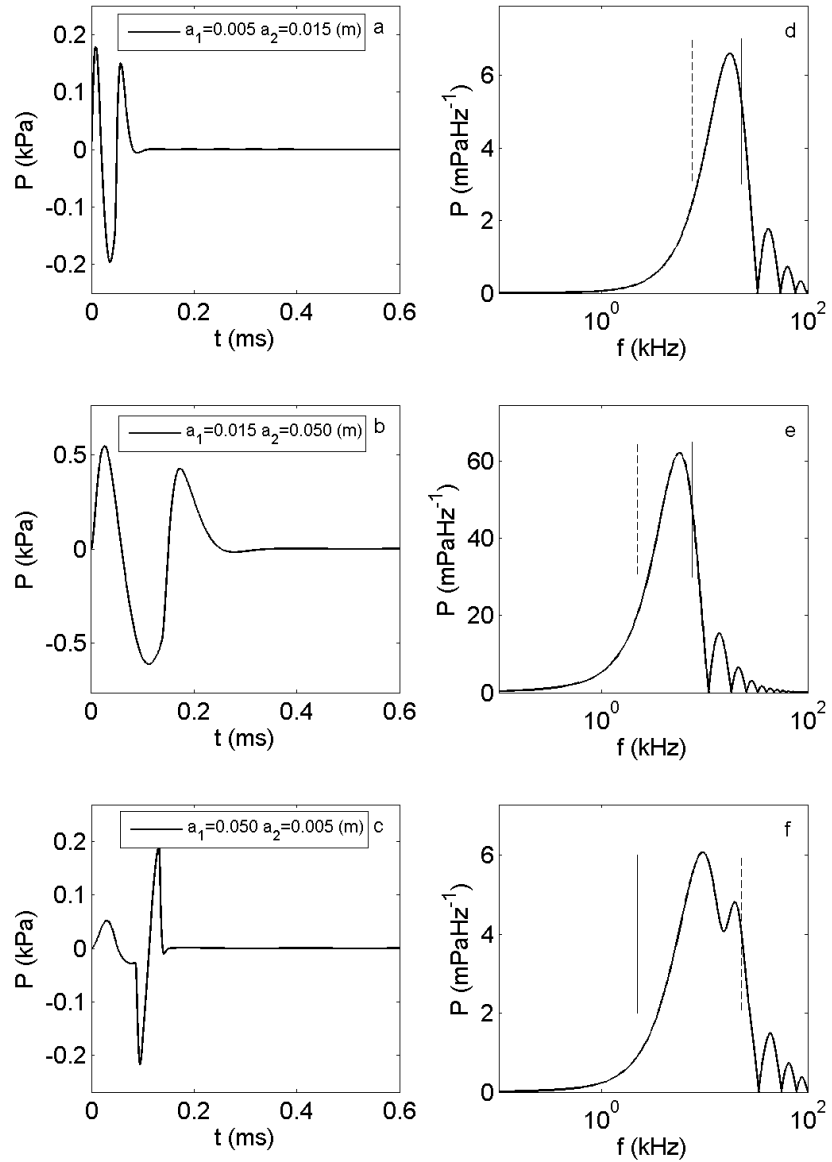

Figure 4. Calculations for glass spheres of different sizes impacting. (a)-(c) Time domain waveform calculated using Eq. (1) and (d)-(f) frequency spectrum calculated using Eq. (2). The vertical lines are the location of the peak frequencies for spheres of equal size impacting have respectively radii of the impactee, $a_{1}(-)$, and the impactor, $a_{2}(--)$.

shows the signal amplitude can be approximated as being proportional to $U / \xi^{2}$, which due to $\xi$ dependence on $t_{0}$ results in a signal amplitude related to $U^{1.4}$. For the changes in $\theta$, the response is observed in plots $5 \mathrm{c}$ and $\mathrm{d}$, where an increase in $\theta$ has the primary response of decreasing the signal amplitude and a second-order effect of reducing the duration of the time domain waveform and broadening the frequency spectrum, although the location of the peak frequency in the spectrum remains essentially unchanged. The significant reduction in signal amplitude is owing to the $\cos (\theta)$ term in $P_{\text {to }}, P_{\text {fo }}$ and $T_{\mathrm{d}}$, which leads to the signal amplitude being dipole in form, reducing approximately as $\cos ^{2}(\theta)$, while the decrease in pulse length and broadening of the spectrum is due to the reduction in $T_{\mathrm{d}}$.

The implications from the recently computed results presented in Figs. 3-5 are that, to first order, the duration of the time domain waveform, the width of the spectrum and the frequency at which the spectrum peaks are principally con- 

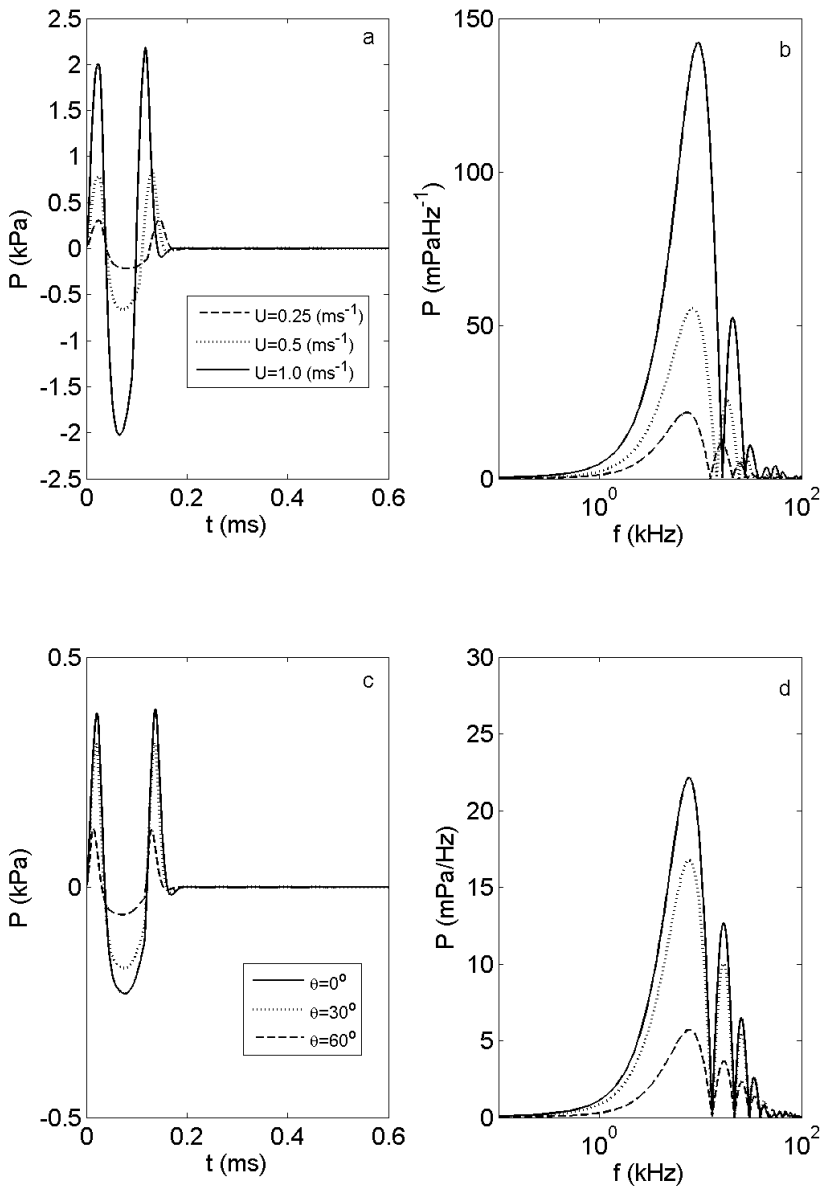

Figure 5. Calculations for the time domain waveform using Eq. (1) and the frequency spectrum using Eq. (2) for $a=0.0015 \mathrm{~m}$ as the velocity, $U$, increased (a and $\mathbf{b}$ ) and as the angle between the direction of impact and the measurement position, $\theta$, increased (c and $\mathbf{d}$ ).

trolled by the size of the spheres impacting, while the amplitude of the time domain waveform and frequency spectrum depend upon sphere size and impact velocity, with the additional factor of the dipole structure for the amplitude of the radiated field.

\section{2 (ii) Multiple particle pair impacts}

Laboratory measurements on multiple particles impacting have been carried out by Jonys (1976), Millard (1976) and Thorne (1985, 1986a). The works of Thorne incorporated the results of the earlier works and are still the most comprehensive study of the underwater sound radiated from multiple collisions of quasi-bedload transport, and therefore the measurements from these two papers are used here to illustrate the salient acoustic features.

The instrumentation used has been previously described (Thorne, 1985); therefore only a brief description is given here. Sediments were agitated in a vertical wooden drum $1 \mathrm{~m}$ in diameter and $0.5 \mathrm{~m}$ deep; this rotated about a horizontal
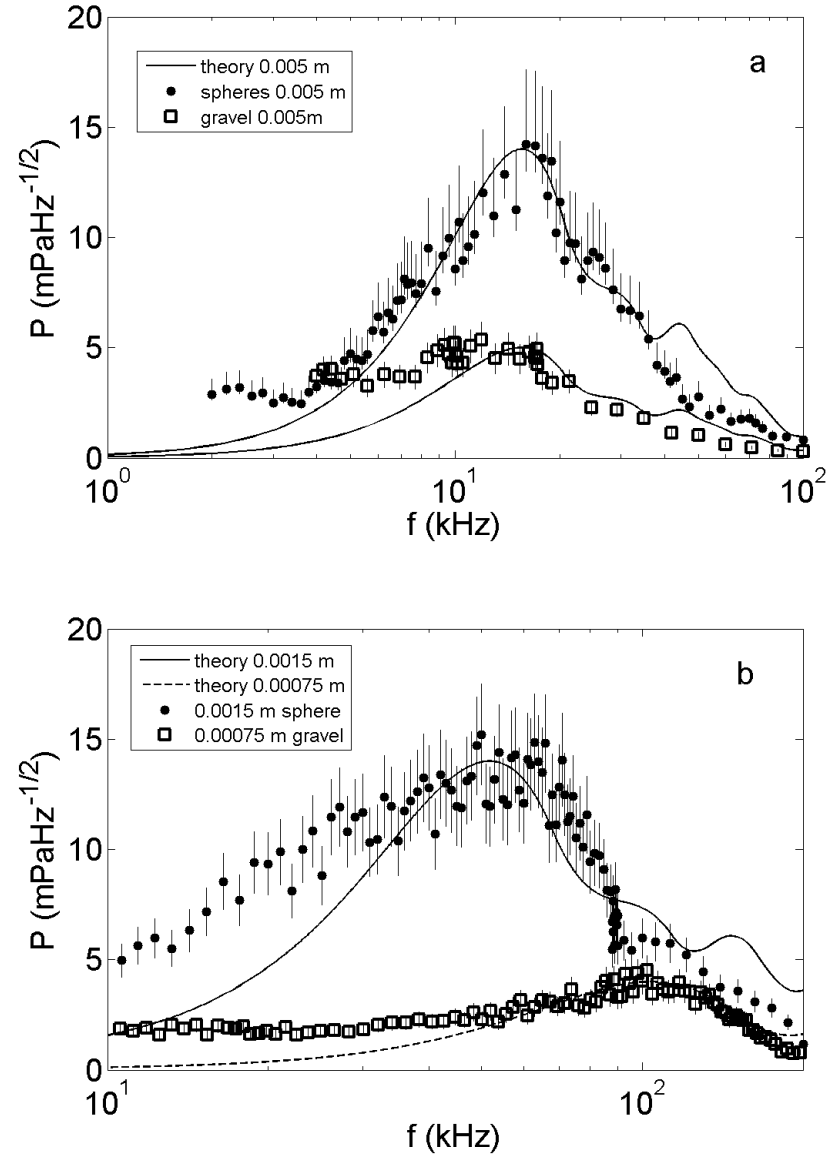

Figure 6. Comparison of the measured and calculated spectra using Eq. (2) with smoothing, for quasi-bedload conditions in a rotating drum for; (a) $0.005 \mathrm{~m}$ radius glass spheres and gravel and (b) $0.0015 \mathrm{~m}$ radius glass spheres and $0.00075 \mathrm{~mm}$ radius gravel.

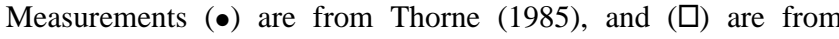
Thorne (1986a).

axis and was totally submerged underwater in a concrete tank $(3 \times 2 \times 2 \mathrm{~m})$. The drum was lined with a $0.002 \mathrm{~m}$ thick expanded polystyrene sheet to ensure that the only significant collision noise generated was due to interparticle collisions. The front of the drum was open, apart from a small lip around the circumference to retain the sediments, thereby allowing hydrophones (underwater sound receivers) to be placed at any position inside the drum while the material was being agitated. The output from the hydrophones was fed into a lownoise amplifier, then through a filter with a passband between $1 \mathrm{kHz}$ and $600 \mathrm{kHz}$, and in parallel into an signal envelope detector and a spectrum analyser. The results were corrected for background noise and the instrumentation frequency response. To account for the fact that measurements were conducted in reverberant tank, rather than the free-field conditions in a riverine environment, measurements were made using a known broadband noise source in the tank (Thorne, 1985; see Appendix). This analysis showed that measure- 

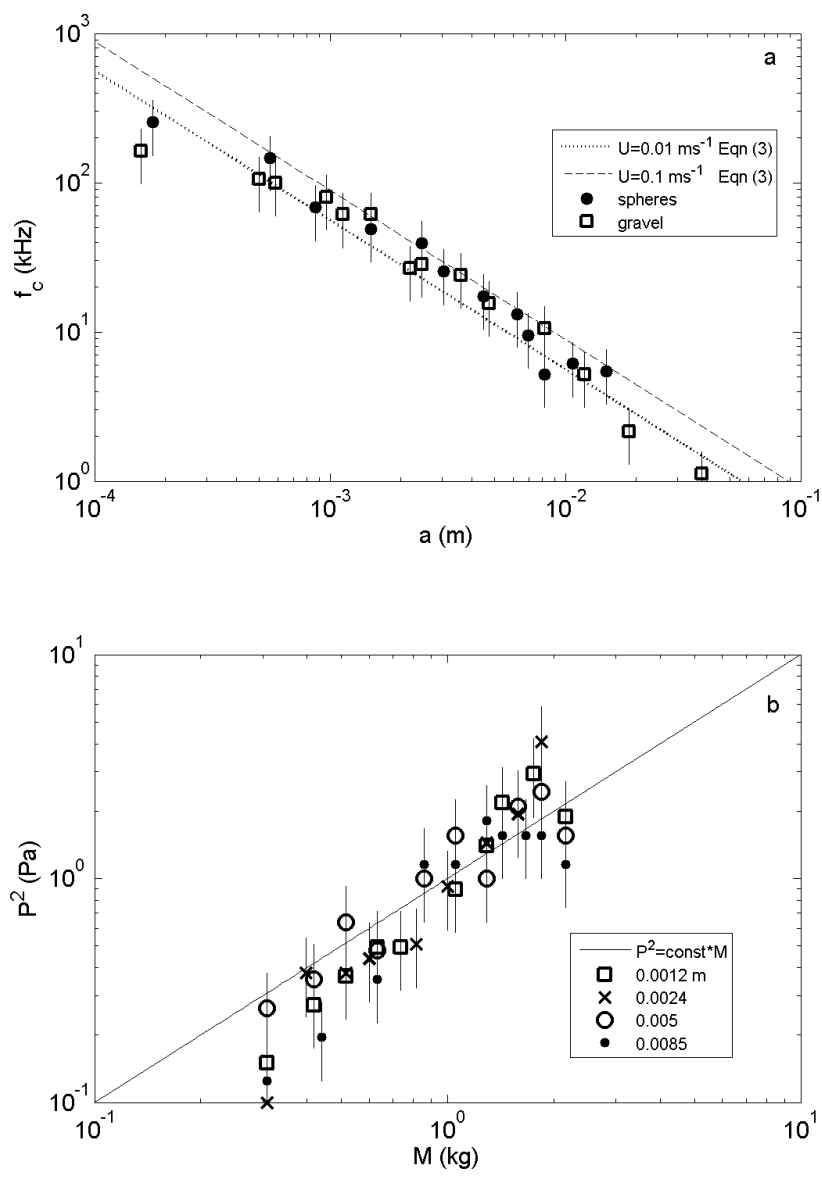

Figure 7. (a) Calculated characteristic central frequency, $f_{\mathrm{c}}$, obtained using Eq. (3) and measurements with particle radius, $a$. (b) The mean square pressure, $P^{2}$, with mass, $M$, compared with Eq. (4). Measurements were collected under quasi-bedload conditions. Measurements are from Jonys (1976), Millard (1976), Thorne (1985) and Thorne (1986a).

ments in a confined reverberant environment can yield effectively free-field amplitude and spectral levels. The measurements in the rotating drum were intended to simulate quasibedload transport and impacts. Measurements were carried out on glass spheres ranging in radius between $a=0.0018$ and $0.015 \mathrm{~m}$, and gravels between $a=0.0015$ and $0.0125 \mathrm{~m}$. In Figs. 6 and 7 some of the measurements are compared with predictions from the rigid body model of acoustic radiation due to impact.

Presented in Fig. 6 are the spectrum for particles centred on nominal radii of $0.00075,0.0015$ and $0.005 \mathrm{~m}$. In Fig. 6a data for spheres and gravel of radius of $0.005 \mathrm{~m}$ are shown; both data sets are broadband in nature, with spectral peaks frequencies being around $15 \mathrm{kHz}$. The fact that spectral levels are lower for the gravel is not necessarily significant because measurements were made under different conditions of mass and rotation speed. Owing to the impact velocities, $U$, the number of particles impacting, $N$, and the value of the angle between the direction of impact and the measurement position, $\theta$, not being well specified in the rotating drum experiments, the output from the rigid body radiation calculations were scaled to the observations. Further using Hertzian impact theory as opposed to non-Hertzian (Riner and Petculescu, 2010) and assuming the particles are spherical as opposed to, say, more cylindrical shape (Wang and Tong, 1992) may be somewhat restrictive for comparisons with the gravel data, however, it provides internal consistency between the sphere and gravel analysis. The comparison is therefore between the modelled and measured spectral form. Further, when multiple particle size pairs of slightly different effective sizes and velocities are impacting simultaneously, marginally different overlapping frequency spectra are summed at the receiver. Therefore for the rigid body calculations the spectra have been smoothed to reduce the oscillations in the spectrum observed in the previous figures associated with only a single size particle pair impacting. This application of rigid body radiation theory to quasi-bedload transport is an important step in acoustic modelling in the riverine environment and the inversion of the acoustic measurements to obtain particle size and bedload transport. As can be seen in Fig. 6a the smoothed rigid body radiation spectra compare reasonably well in form with the sphere and gravel spectra, although, particularly for the gravel, the reduction in the spectral amplitude at lower frequencies seen in the data is smaller than predicted by the model. This was a common and unresolved observation for gravel across most of the measurements (Thorne 1986a, 1990). In Fig. 6b, results for $0.0015 \mathrm{~m}$ glass spheres and $0.00075 \mathrm{~m}$ gravel are shown, and the measurements clearly show a separation in the spectra, with the smaller gravel material having a maximum in the spectrum at twice the frequency of that of the glass spheres. Comparison of the predicted spectrum with the observations again captures the broadband nature of the sound, the location of the maximum in the spectrum, the rolloff at the higher frequencies and the smaller material having a maximum amplitude at a higher frequency; however, as with the cases in Fig. 6a, the modelled lower frequency components are underestimated.

To represent the variation of the spectra with particle size, the frequency at which the spectrum nominally peaks, or the centroid of the spectrum, has been used to define a characteristic central frequency. This is illustrated in Fig. 7a, where results from the studies of Jonys (1976), Millard (1976) and Thorne $(1985,1986 a)$ are presented. What can be clearly seen is that the characteristic central frequency has to first order an inverse dependency on particle size. From rigid body radiation theory the frequency at which the spectrum peaks, $f_{\mathrm{pk}}$, can be seen in Fig. 2b to be approximately given by $1.7 f_{\mathrm{o}}$, where $f_{\mathrm{o}}=1 / 2 t_{\mathrm{o}}$. For simplicity, if the assumption is made that two identical particles are impacting, the expression for $t_{\mathrm{o}}$ can be simplified (see the definition below Eq. 1), 
and this allows $f_{\mathrm{pk}}$ to be expressed as

$f_{\mathrm{pk}}=0.15\left\{\frac{E}{\rho_{\mathrm{s}}\left(1-\sigma^{2}\right)}\right\}^{0.4} \frac{U^{0.2}}{a}$

All the parameters in Eq. (3) were known apart from the particle impact velocities in the rotating drum. Typical circumferential drum speeds of $0.3 \mathrm{~ms}^{-1}$ were used so impact velocities in the range of $0.01-0.1 \mathrm{~ms}^{-1}$ would not seem unreasonable and were therefore used in the evaluation of Eq. (3) to obtain the lines in Fig. 7a. The data generally lie between the two lines, thereby indicating that Eq. (3) is probably a reasonable description for a characteristic central frequency for the broadband impact spectrum, with, guided by Fig. 2b, the significant section of the spectrum lying in the frequency band between approximately $f_{\mathrm{pk}} / 4$ and $4 f_{\mathrm{pk}}$.

In the marine environment the amount of material transported as bedload will vary over time depending on the size of the sediments on the bed and the hydrodynamic conditions. To simulate this variability, a series of measurement were carried out on gravels of different radii, with $a=0.0012,0.0024,0.005$ and $0.0085 \mathrm{~m}$ where the mass, $M$, of sediments in the drum was increased at constant rotation speed. Treating the interparticle impacts in the drum as similar random, independent noise sources, the total signal can be expressed as (Beranek, 1971)

$P^{2}=\sum_{i=1}^{N} P_{i}^{2} \approx N \overline{P_{i}^{2}}$

where $N$ is the number of sources, $P_{i}^{2}$ are the individual source pressure levels squared, the over-bar represents a mean value and $P^{2}$ is the total mean squared pressure. As $N$ is proportional to $M, P^{2}$ should be approximately linearly dependent on $M$. Figure $7 \mathrm{~b}$ shows the results of the measurements and the line represents Eq. (4). Although there is some variability in the four data sets, the general slope of the data is consistent with a linear relationship between $P^{2}$ and $M$. The scatter in the data is associated with variable impact velocities about a mean, the different size particles within a samples and non-sphericity of the particles impacting.

In general therefore, it can be seen that the relatively simple rigid body radiation model captures in broad terms the form of the spectrum for large numbers of particles impacting in a quasi-bedload manner, although for reasons not resolved in the present analysis the lower frequency components are somewhat underpredicted. The spectrum can be broadly specified as having a characteristic central frequency which is inversely related to particle size and with a bandwidth nominally between $f_{\mathrm{pk}} / 4$ and $4 f_{\mathrm{pk}}$. Finally to represent increases in bedload, the amount of material in the drum was gradually increased at a constant rotation speed, which resulted in the mean squared pressure increasing linearly with mass. These results do indicate that there is the possibility that inversion algorithms could be developed in
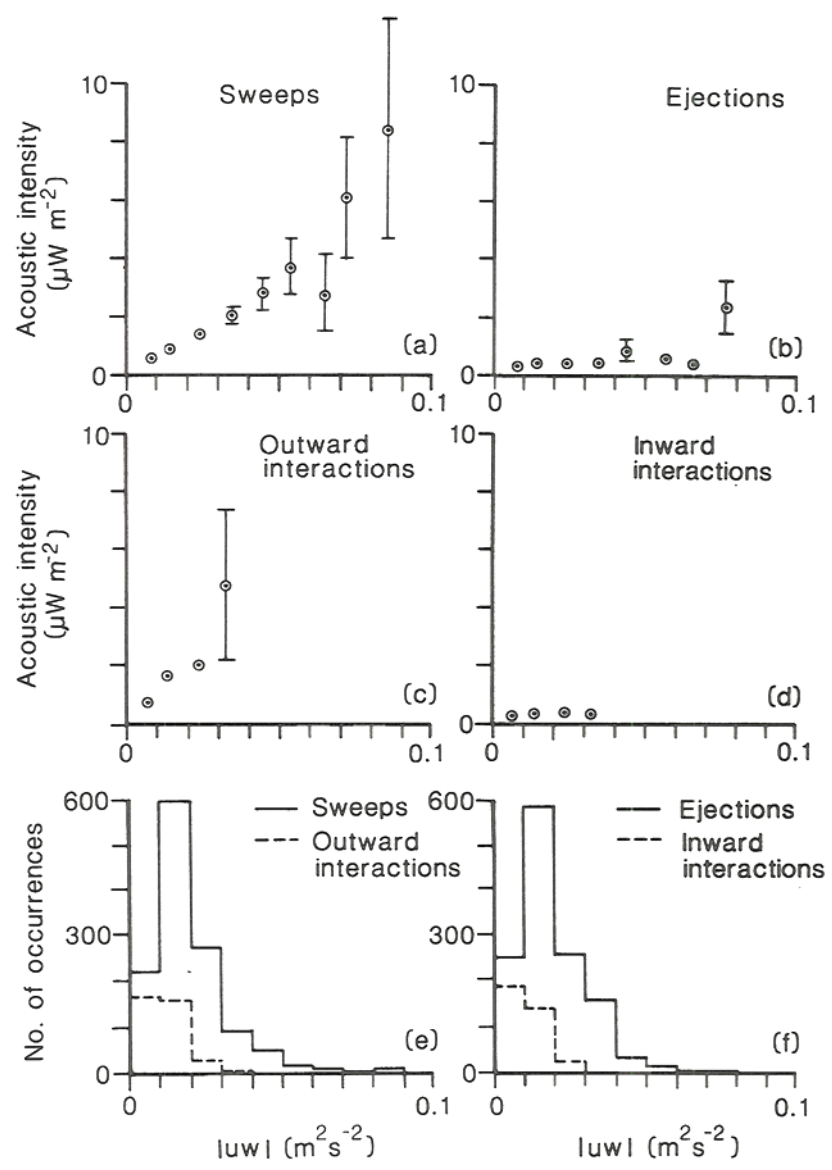

Figure 8. Comparison of the proxy for sediment transport, acoustic intensity, with the magnitude of the kinematic stress, $|\mathrm{uw}|$, due to (a) sweeps, (b) ejections, (c) outward interactions, (d) inward interactions and $(\mathbf{e}, \mathbf{f})$ the number of events for the four kinematic stress quadrature components. Measurements are from Heathershaw and Thorne (1985).

the future for estimating bedload parameters from the signature of the SGN signal.

\section{Example from a field study}

There have been a number of field trials of the SGN technique, and the results have been variable (Bedeus and Ivicsics, 1963; Tywoniuk and Warnock, 1973; Jonys 1976; Richards and Milne, 1979; Thorne et al., 1989; Williams et al., 1989; Voulgaris et al., 1995; Mason et al., 2007; Barton et al., 2010; Belleudy, 2010; Bassett et al., 2013). One of the more successful and interesting studies was to utilise the non-intrusive high-temporal-resolution measuring capability of SGN to examine the relationship between turbulent bursting in tidal flows and the bedload transport of coarse gravels (Heathershaw and Thorne, 1985; Thorne et al., 1989). Using an instrumented frame, concurrent measurements of the three orthogonal components of the turbulent flow and in- 
stantaneous bedload transport were collected above a gravel bed in a tidally dynamic environment with currents peaking at around $1.0 \mathrm{~ms}^{-1}$ at $1 \mathrm{~m}$ above the bed. High-resolution bedload transport measurements were derived from two hydrophones measuring the SGN and calibrated in situ for bedload transport using visual measurements of sediment transport using an underwater video camera (Thorne, 1986b). The flow and acoustic measurements were respectively collected at 0.33 and $0.24 \mathrm{~m}$ above the bed, and the synchronised data were digitised at $5.0 \mathrm{~Hz}$ and recorded. From the turbulent flow measurements the kinematic Reynolds stress, $\tau_{\mathrm{uw}}$, was calculate using $\tau_{\mathrm{uw}}=-\mathrm{uw} \mathrm{m} \mathrm{m}^{2} \mathrm{~s}^{-2}$, where $\mathrm{u}$ and $\mathrm{w}$ were respectively the horizontal and vertical fluctuating turbulent components of the flow. From the acoustic calibration it was shown that the SGN acoustic intensity, $I \mu \mathrm{Wm}^{-2}$, which is proportional to the squared pressure, was an acceptable surrogate for bedload transport (Thorne, 1986b). Applying a quadrature analysis to the kinematic Reynolds stress, events in the flow were identified as sweeps $(u>0, w<0)$, ejections $(\mathrm{u}<0, \mathrm{w}>0)$, outward $(\mathrm{u}>0, \mathrm{w}>0)$ and inward $(\mathrm{u}<0$, $\mathrm{w}<0$ ) interactions and compared with the corresponding values for $I$. The results of the analysis are shown in Fig. 8.

The measurements demonstrated quite clearly in Fig. 8a and $b$ that the acoustic intensity, and hence gravel transport, associated with sweep events were substantially higher than the intensity levels during ejection events at high stress values. This difference increased as the magnitude of the kinematic stress increased. From this it was concluded that of the two types of motion that contributed to the bulk of the kinematic Reynolds stress, ejections and sweeps, only sweeps were capable of supporting appreciable coarse sediment movement. It was also noted that unexpectedly outward interaction events, although weaker and less frequent than sweeps, as shown in Fig. 8c and e, were capable of supporting greater sediment movement than sweeps for the same stress levels. This is despite the fact that they make a negative contribution to the Reynolds stress. Correspondingly, there was little sediment movement associated with inward interactions. The results showed that horizontal turbulent velocity fluctuations, $\mathrm{u}$, may have greater dynamical significance in terms of coarse sediment movement than the instantaneous contributions, - uw, to the Reynolds stress. Also vertical fluctuations were considered as important provided they were associated with increases in $u$. This was the case for outward interactions, Fig. 3c, where $\mathrm{w}>0$ indicated additional lift on exposed gravel particles by fluid moving away from the bed. This, in turn was considered to account for sediment transport rates being higher than those of similarly sized sweeps. The observed close dependence on $\mathrm{u}$ was considered explainable if the gravel were moved principally by form drag acting on the flow-normal projected area of exposed particles. To examine this, a correlation analysis between the total horizontal flow, $U$, and $I$, and Reynolds stress, $-\rho_{\mathrm{o}} \overline{u w}$, and $I$ were carried out. The results of the analysis showed that in all cases sediment movement was better correlated with form drag than with the instantaneous stress.

This study illustrated that SGN can provide detailed hightemporal-resolution measurements of sediment response to turbulent flow conditions and showed for the first time that the bedload movement of seabed gravels is caused principally by sweep-type motions in the bottom boundary layer and to a lesser extent by outward interactions. This observation could be explained if form drag rather than shear stress were assumed to be the principle cause of gravel movement. It was speculated that such relationships between sediment transport and turbulent motions could lead to a new generation of sediment transport equations which accounted for the turbulent bursting process (Clifford et al., 1995; Williams, 1996; Sumer et al., 2003).

\section{Discussion and conclusion}

The aim of the present paper has been to provide scientist and engineers who are interested in the measurement of coarse sediment bedload transport with an overview of the background to sediment-generated noise. The new calculations presented here on sphere impacts and comparisons with quasi-bedload transport have illustrated that the rigid body radiation approach should provide a first-order framework for understanding and interpreting SGN collected in riverine and coastal environments. When the bed becomes mobile, interparticle collisions occur which radiate sound into the water, and this SGN can been used as a proxy for bedload transport rates. To understand and predict the sound field generated by the collision of particles, a theoretical framework based on rigid body radiation has been presented. Initially predictions were made with colliding pairs of glass spheres, and the impact of sphere size, impact velocity and field point angle was examined to assess the effect these had on the measured time domain signal and the frequency spectrum. Limited comparison with available data was carried out to assess the veracity of the theory. To move beyond simple two-particle impacts, larger numbers of particles were impacted using a rotating drum arrangement; this experimental configuration was employed to simulate quasi-bedload conditions. In these studies data were collected on glass spheres and natural gravels. Spectral analysis of the measurements showed comparable spectra to the two sphere impact results, and rigid body radiation gave reasonable first-order agreement with the rotating drum data. Assessment of a characteristic central frequency for the spectra showed a clear inverse relationship with the size of the impacting particles, and an expression derived from the impact duration of the collision time, $t_{\mathrm{o}}$, given by Eq. (3), provided a reasonable description for the observations. To establish a relationship between the amount of material impacting in the rotating drum and the mean square signal level recorded, measurement were carried out for a number of different particle sizes 
at constant drum rotation speed. The results showed that the mean square signal level was proportional to the amount of material in the drum and hence ostensibly the number of collisions. To explain the observations, the interparticle impacts in the drum were considered to be similar random independent noise sources which summed linearly with the mean square pressure.

The outcome from the two sphere impact studies and the measurements in the rotating drum indicated that the relatively simple Eqs. (1)-(4), derived from rigid body radiation, and the linear summation of mean square pressures, provide a framework for a first-order understanding of SGN. The results showed that, for pairs of spheres impacting, the amplitude of the signal is a function of the sphere size, impact velocity and the location of the position of observation. For measurements of bedload in the field, the location of the receiver will normally remain fixed relative to the bed and the size of the bedload material will be nominally constant; therefore the signal amplitude will essentially depend on the impact velocity and the number of particles impacting. If it is assumed that impact velocity is proportional to the velocity on the mobile material, then the mean square signal amplitude should be acting ostensibly as a nominal proxy for the bedload transport, and this has been reported in a number of studies (Johnson and Muir, 1969; Thorne, 1986b; Barton et al., 2010). The form of the spectrum has been shown to be primarily dependent on the size of the impacting particles, with the impact velocity and measurement location having only second-order effects. The form of the spectrum is therefore a reasonably robust indicator of the size of the mobile material and as such has been used to estimate the size of the bedload material (Thorne, 1986a; Mason et al., 2007; Belleudy et al., 2010; Basset et al., 2013).
One of the more common difficulties in the application of SGN to the measurement of coarse sediment bedload transport is the level of the background aquatic soundscape (Wenz, 1972; Thorne, 1986b; Vracar and Mijic, 2011). Contributions from biophony (sounds from aquatic animals), geophony (sounds from natural abiotic phenomenon) and anthrophony (sounds from manmade activities) can make interpretation and assessment of the SGN problematic. To-date most SGN measurements have been collected using nominally omnidirectional hydrophones. Looking to the future, the mounting of such hydrophones in acoustically reflective housings to increase directionality (as with an omnidirectional bulb in a car headlight), and thereby rejecting erroneous background noise, could be an interesting step forward. Also given that predictions can be made for the spectrum of the sound from a knowledge of particle size, this may be used with bandpass filtering to enhance the SGN signal relative to the general soundscape. One area which is still deficient is rigorous assessments of the SGN technique using independent measurements of coarse sediment bedload transport. Further studies in flumes and in the field would establish, with greater veracity than available at present, the capabilities and uncertainties in the application of SGN to the robust measurement of bedload transport and particle size. 
Appendix A

Table A1. List of variables.

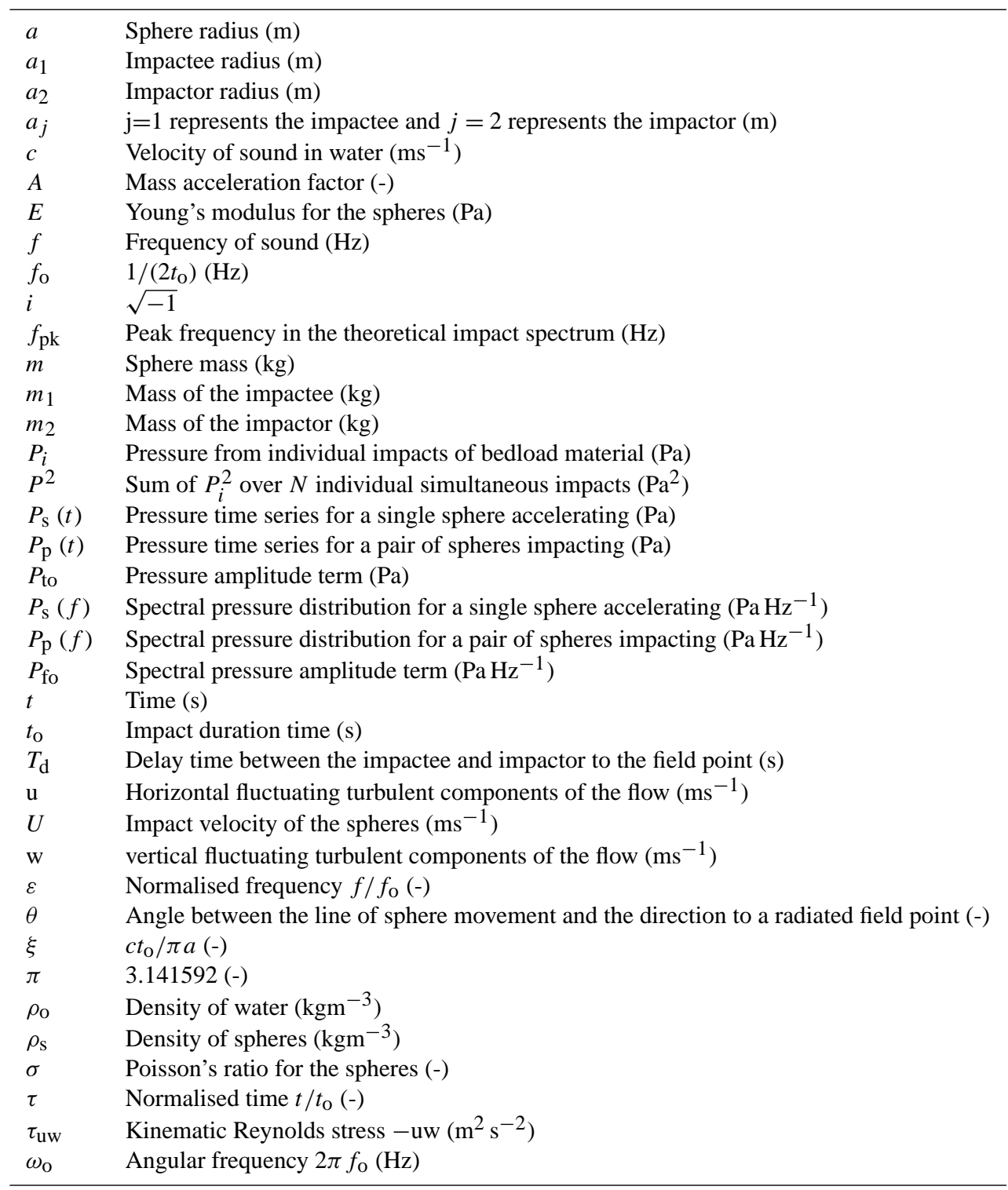


Acknowledgements. This overview on sediment-generated noise and coarse bedload transport originated from an invitation to the author to provide a keynote presentation at the International Workshop of Acoustic and Seismic Monitoring of Bedload and Mass Movements held in Zurich, Switzerland, 4-7 September 2013. The author thanks the organisers of the workshop and in particular Jonathan Laronne for the invitation. The preparation of this manuscript was carried out following an invite to make a submission to a special issue section on "Acoustic and seismic monitoring of bedload and mass movements" as part of the journal Earth Surface Dynamics. The study was supported by funding from the Natural Environmental Research Council, UK, National Capability.

Edited by: J. Turowski

\section{References}

Akay, A.: A review of impact noise, J. Acoust. Soc. Am., 64, 977987, 1978.

Akay, A. and Hodgson, T. H.: Acoustic radiation from the elastic impact of a sphere with a slab, Appl. Acoust., 11, 185-304, 1978a.

Akay, A. and Hodgson, T. H.: Sound radiation from an accelerated or decelerated sphere, J. Acoust. Soc. Am. 63, 313-318, 1978b.

Banerji, S.: On aerial waves generated by impact, Philos. Mag. J. Sci., 6.32, 96-111, 1916.

Banerji, S.: On aerial waves generated by impact, Part II, Philos. Mag. J. Sci., 6.35, 97-111, 1918.

Barton, J. S., Slingerland, R. L., Pittman, S., and Gabrielson, T. B.: Monitoring coarse bedload transport with passive acoustic instrumentation: A field study. Published online in 2010 by USGS as part of US Geological Survey Scientific Investigations Report 2010-5091, available at: http://pubs.usgs.gov/sir/2010/5091/ papers/listofpapers.html, 38-51, 2010.

Bassett, C., Thomson, J., and Polagye, B.: Sediment-generated noise and bed stress in a tidal channel, J. Geophys. Res.-Oceans, 118, 2249-2265, doi:10.1002/jgrc.20169, 2013.

Bedeus, K. and Iviscics, L.: Observations of the noise of bedload, Proc. Intl. Assoc. Sci. Hydrol., 65, 384-390, 1963.

Belleudy, P., Valette, A., and Graff, B.: Passive hydrophone monitoring of bedload in river beds: First trials of signal spectral analysis. Published online in 2010 by USGS as part of US Geological Survey Scientific Investigations Report 2010-5091, available at: http://pubs.usgs.gov/sir/2010/5091/papers/listofpapers.html, 6784, 2010.

Beranek, L. L.: Noise and Vibration Control, Published by Mcgrawhill, New York, Chap. 2, 1971.

Bunte, K., Abt, S. R., Potyondy, J. P., and Swingle, K. W.: A Comparison of Coarse Bedload Transport Measured with Bedload Traps and Helley-Smith Samplers, Geodinamica Acta, 21/1-2, 53-66, 2008.

Burtin, A., Cattin, R., Bollinger, L., Vergne, J., Steer, P., Robert, A., Findling, N., and Tiberi, C.: Towards the hydrologic and bed load monitoring from high-frequency seismic noise in a braided river: The 'torrent de St Pierre', French Alps, J. Hydrol., 408, 43-53, 2011.
Camenen, B., Jaballah, M., Geay, T., Belleudy, P., Laronne ,J. B., and Laskowski, J. P.: Tentative measurements of bedload transport in an energetic alpine gravel bed river, in: River flow 2012 edited by: Murillo, R., Taylor and Francis Group London, 379386, ISBN 978 0-415-62129-8, 2012.

Clifford, N. J., Richards, R. A., Brown, R. A., and Lane, S. N.: Scales of variation of suspended sediment concentration and turbidity in a glacial meltwater stream, Geograf. Annal., 77A, 4565, 1995.

Crickmore, M. J., Waters, C. B., and Price, W. A.: The measurement of offshore shingle movement. Proceedings of the Thirteenth Coastal Engineering Conference held at Vancouver, Canada, 2, 1005-1025, 1972.

Dorey, A. P., Finch, A. R., and Dyer, K. R.: A miniature transponding pebble for studying gravel movement. In Conference proceedings on Instrumentation in Oceanography held at University College of North Wales, Bangor, 327-332, 1975.

Ergenzinger, P. and de Jong, C.: Perspectives on bed load measurement, in: Erosion and sediment transport measurement in rivers: Technological and methodological advances (Proceedings of the Oslo Workshop, June 2002), IAHS Publ. 283, 113-125, 2003.

Engel, P. and Lam Lau, Y.: The efficiency of basket type bedload samplers. Erosion and sediment transport measurements. Proc of the Florence Symposium, IAHR Publ., 133, 27-34, 1981

Goldsmith, W.: Impact, Impact Published by Edward Arnold, London, Chap. 4, 1960.

Gray, J. R., Laronne, J. B., and Marr, J. D. G.: Surrogate Bedload Monitoring Techniques, US Geological Survey Scientific Investigations Report 2010-5091, 37 p., available at: http://pubs.usgs. gov/sir/2010/5091, The 26 included papers are available at: http: //pubs.usgs.gov/sir/2010/5091/papers/listofpapers.html, 2010.

Heathershaw, A. D. and Thorne, P. D.: Sea-bed noises reveal role of turbulent bursting phenomenon in sediment transport by tidal currents, Nature, 316, 339-342, 1985.

Holmes, R. R.: Measurement of Bedload Transport in Sand-Bed Rivers: A Look at Two Indirect Sampling Methods. Published online in 2010 by USGS as part of US Geological Survey Scientific Investigations Report 2010-5091, available at: http:// pubs.usgs.gov/sir/2010/5091/papers/listofpapers.html, 236-252, 2010.

Hubbell, D. W.: Apparatus and techniques for measuring bedload, paper N 182/B/1748, US Geological Survey Water-Supply, US Geological Survey, Washington, 1-74, 1964.

Johnson, P. and Muir, T. C.: Acoustic detection of sediment movement, J. Hydraul. Res., 7, 519-540, 1969.

Jonys, C. K.: Acoustic measurement of sediment transport, Scientific Series no. 66, Department of Fisheries and the Environment, Inland Waters Directorate CCIW Branch, Burlington, Ontario, Canada, 1-114, 1976.

Kaye, G. W. C. and Laby, T. H.: Tables of Physical and Chemical Constants, Published by Longman, New York, 15th Edn., 1986.

Koss, L. L.: Transient sound from colliding spheres - inelastic collisions, J. Sound Vib., 36, 555-562, 1974a.

Koss, L. L.: Transient sound from colliding spheres - Normalized results, J. Sound Vib., 36, 541-553, 1974b.

Koss, L. L. and Alfredson, R. J.: Transient sound radiated by spheres undergoing an elastic collision, J. Sound Vib., 27, 59$75,1973$. 
Mason, T., Priestley, D., and Reeve, D. E.: Monitoring near shore shingle transport under waves using a passive acoustic technique, J. Acoust. Soc. Am., 122, 737-746, doi:10.1121/1.2747196, 2007.

Millard, N. W.: Noise generated by moving sediments. Proceedings of Conference on Recent Developments in Underwater Acoustics, Institute of Acoustics, Held at AUWE, Portland, 31 March1 April, paper 3.5, 1976.

Reid, I., Brayshaw, A. C., and Frostick, L. E.: An electromagnetic device for automatic detection of bedload motion and its field application, Sedimentology, 31, 269-276, 1984.

Rennie, C. D. and Church, M.: Mapping spatial distributions and uncertainty of water and sediment flux in a large gravel bed river reach using an acoustic Doppler current profiler, J. Geophys. Res., 115, F03035, doi:10.1029/2009JF001556, 2010.

Richards, K. S. and Milne, L. M.: Problems in the calibration of an acoustic device for the observation of bedload transport, Earth Surf. Process., 4, 335-346, 1979.

Rickenmann, D., Turowski, J., Fritschi, B., Klaiber, A., and Bedload, L. A.: transport measurements at the Erlenbach stream with geophone sand automated basket samplers, Earth Surf. Process. Landf., 37, 1000-1011, 2012.

Rickenmann, D., Laronne, J. B., Turowski, J. M., and Vericat, D.: International Workshop of Acoustic and Seismic Monitoring of Bedload and Mass Movements, 4-7 September, Birmensdorf, Zürich, Switzerland, Abstracts, available at: http://www.wsl.ch/ dienstleistungen/publikationen/pdf/12904.pdf, 2013.

Riner, J. and Petculescu, A.: Non-Hertzian behavior in binary collisions of plastic balls derived from impact acoustics, J. Acoust. Soc. Am., 128, 132-136, 2010

Sumer, B. M., Chua, L. H. C., Cheng, N. S., and Fredsoe, J.: Influence of turbulaence on bed load sediment transport, J. Hydraul. Eng., 12, 585-596, 2003.

Thorne, P. D.: The measurement of acoustic noise generated by moving artificial sediments, J. Acoust. Soc. Am., 78, 1013-1023, 1985.

Thorne, P. D.: Laboratory and marine measurements on the acoustic detection of sediment transport, J. Acoust. Soc. Ame., 80, 899910,1986 .
Thorne, P. D.: An intercomparison between visual and acoustic detection of seabed gravel movements, Mar. Geol., 72, 11-31, $1986 b$.

Thorne, P. D.: Seabed generation of ambient noise, J. Acoust. Soc. Am., 87, 149-153, 1990.

Thorne, P. D. and Foden, D. J.: Generation of underwater sound by colliding spheres, J. Acoust. Soc. Am., 84, 2144-2152, 1988.

Thorne, P. D., Heathershaw, A. D., and Troiano, L.: Acoustic detection of seabed gravel movement in turbulent tidal currents, Mar. Geol., 54, M43-M48, 1984.

Thorne, P. D., Williams, J. J., and Heathershaw, A. D.: In situ acoustic measurements of marine gravel thereshold and transport, Sedimentology, 36, 61-74, 1989.

Tsai, V. C., Minchew, B., Lamb, M. P., and Ampuero, J. P.: A physical model for seismic noise generation from sediment transport in rivers, Geophys. Res. Lett., 39, L02404, doi:10.1029/2011GL050255, 2012.

Turowski, J. M. and Rickenmann, D.: Tools and cover effects in bedload transport observations in the Pitzbach, Austria, Earth Surf. Process. Landf., 34, 26-37, 2009.

Tywoniuk, N. and Warnock, R. G.: Acoustic detection of bedload transport. Proceedings of the 9th Canadian Hydrology Symposium, Ottawa, Ontario, Canada, 728-749, 1973.

Voulgaris, G., Wilkin, M. P., and Collins, M. B.: The in situ passive acoustic measurement of shingle movement under waves and currents: instrument (TOSCA) development and preliminary results, Continental Shelf Res., 15, 1195-1211, 1995.

Vracar, M. S. and Mijic, M.: Ambient noise in large rivers, J. Acoust. Soc. Am., 130, 1787-1791, 2011.

Wang, Y. F. and Tong, Z. F.: Sound radiated from the impact of two cylinders, J. Sound Vibration, 159, 295-303, 1992.

Wenz, G. M.: Review of underwater acoustic research:Noise, J. Acoust. Soc. Am., 51, 3, 1010-1024, 1972.

William, J. J.: Turbulent flow in rivers, in: Advances in Fluvial Dynamics and Stratigraphy. edited by: Carling, P. A. and Dawson, M. R., Published by John Wiley and Sons Ltd., 1-32, 1996.

Williams, J. J., Thorne, P. D., and Heathershaw, A. D.: Comparison between acoustic measurements and predictions of the bedload transport of marine gravels, Sedimentology, 36, 973-979, 1989. 